\title{
The Future of Diffraction at Tevatron
}

\author{
Alberto Santoro ${ }^{\mathrm{a}}$ \\ ${ }^{a}$ Centro Brasileiro de Pesquisas Físicas, \\ Rua Dr. Xavier Sigaud, 150 RJ - Rio de Janeiro, Brasil \\ e-mail: santoro@cbpf.br
}

This paper summarizes the main current upgrade for Diffractive Physics at Tevatron. We describe both CDF (Collider Detector Facility) and DØ new devices that are being installed around the CDF and D $\varnothing$ area that will allow to produce new results in Diffractive Physics.

\section{Introduction}

Diffraction is certainly one of the oldest subject of physics. In Particle Physics we have seen a big development in the second half of this century [1]. More recently with the discovery of Hard Diffraction by UA8-Collaboration [2], we have had a significant development of the Diffractive Physics from both theoretical and experimental point of view. This is demonstrated in this workshop with many results coming from Hera (H1 and Zeus) [3] in a large number of topics including diffractive structure functions, and at the Tevatron (CDF and $\mathrm{D} \varnothing)$ with results on diffractively produced jets at both energies $\sqrt{s}=1.8 \mathrm{TeV}$ and $630 \mathrm{GeV}$ [5]. From the theoretical side we had many presentations showing a great number of ideas about diffraction. These results are important to understand the nature of the Pomeron.

All the above experimental results constitute the data sample available for the development of theoretical and phenomenological models allowing the comparison with these predictions. However many results have poor statistics and new data is needed. Some of the processes under study still have not been directly observed also. Information about $t$ and $\xi$ distributions is missing to complete some studies and verify some theoretical results. Despite of the theoretical progress for the unification of the soft and hard aspects of the strong interactions, we need to get new experimental results to exploit deeply theoretical ideas. We have to stress again that $40 \%$ of the cross section is due to the Pomeron exchange, and at higher energy can still be growing. This means that we have to pay attention to this physical region of the proton anti-proton interaction at the Tevatron. We need more data to answer questions like: universality of the Pomeron, hadronic characteristic, is the Pomeron a glueball?, is the Pomeron a dual object?, What is the real contribution for the cross sections of the diffractive heavy flavor production?, Can Higgs and Centauros can be produced at Tevatron energies? What is the actual value of the $\bar{p} p$ total cross section? and so on.

All these questions lead to the proposal of new devices to be inserted at Tevatron, Roman Pots Spectrometer + Miniplug +Beam Shower Counters in the CDF area and a Forward Proton Detector (FPD) for the $\mathrm{D} \varnothing$ area.

Figure 1 shows the growing interest for diffractive physics. There is a clear growing number of papers about this subject in particle physics.

We expect soon a new period of diffractive results from several experiments. From the Tevatron, the next Run II with CDF and D $\varnothing$; from Brookhaven National Laboratory (BNL), Relativistic Heavy Ion Collider (RHIC) in proton proton interactions at the energy of $\sqrt{s}=50-500$ $\mathrm{GeV}$; from the Deutsches Elecktronen Synchroton (DESY), with H1 and ZEUS the two detectors at HERA in electron $(30 \mathrm{GeV})$ proton $(800 \mathrm{GeV})$ interactions. For the near future we will have a new era of energy experiments, with TOTEM [6] being integrated to Compact Muon Solenoid (CMS) 


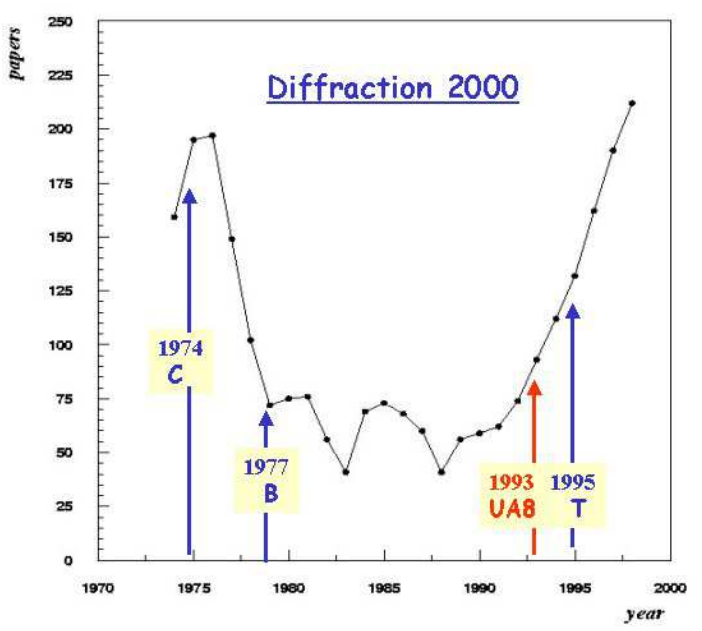

Figure 1. Number of papers per year in diffractive physics 4. We show four important dates which show some correlation with diffractive physics papers. 1974 was the year of charm discovery, three years later, in 1977 the bottom, in 1993 it was the year of the discovery of hard diffraction and 1995 the top was discovery.

as one of the detectors of Large Hadron Collider (LHC) of Centre European for Particle Physics (CERN) for proton proton at $14 \mathrm{TeV}$ energy in center of mass system.

\section{The Tevatron and the upgrades of CDF and $\mathrm{D} \emptyset$ for Diffractive Physics}

The Tevatron itself is being submitted to an upgrade in several parts of the machine. The main parameters for physics are the energy going to $\sqrt{s}$ $=2$. TeV and the higher luminosity. The two detectors CDF and $\mathrm{D} \varnothing$ are also being submitted to many upgrades. We will comment mainly those that have a direct relation with diffractive physics.

The Tevatron beam line was modified to open space for the FPD stations. The Electrostatic separator girder had to be modified, the cryogenic bypass was expanded to open space to allow the insertion of the Roman Pots of FPD, the quadrupole magnet $Q_{1}$ is no longer present on the beam line. Other small modifications were neces- sary, like drilling a hole on the floor to allow the insertion and removal of the bottom detectors. Summarizing the Tevatron modification are: (i) girder modification, (ii) cryogenic bypass, (iii) removal of $Q_{1}$ quadrupole.

\subsection{CDF}

We will summarize the upgrade for diffractive physics for CDF since the reference [7] shows a detailed description of this detector. The CDF upgrade for diffractive physics can be seen in three parts:

1. Improve dipole spectrometer (Roman Pots) as is shown in figure 2

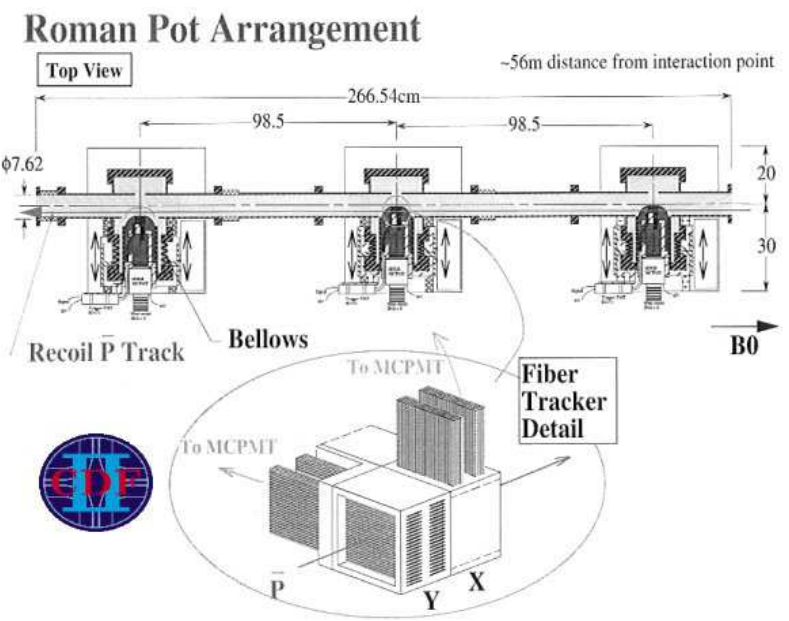

Figure 2. Roman Pots arrangement of CDF.

2. Use the miniplug for triggering Diffractive events. This device is shown in figure 3. Each Miniplug is composed by 50 lead plates of $1 / 4$ thick corresponding to a total of 2 interactions lengths and 60 radiation length. 288 signal towers viewed by 18 MCPMTs (16 channels each) and a total weight of 0.8 tons.

3. Add new scintillating counters at large rapidity 


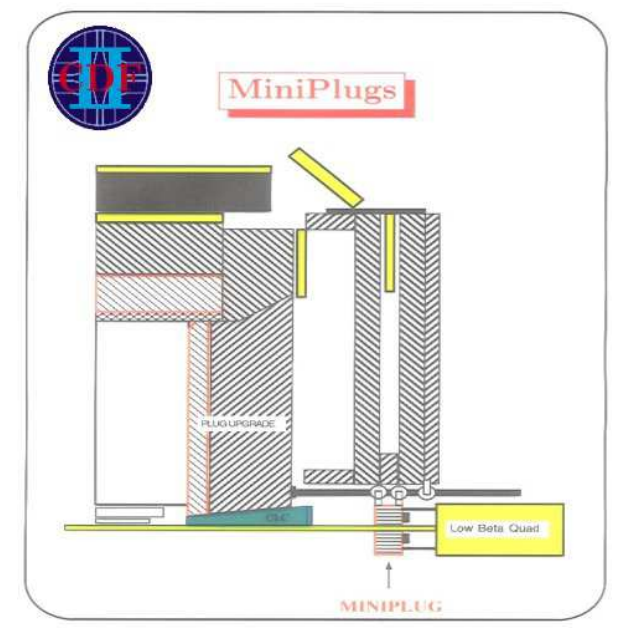

Figure 3. This is the Miniplug of CDF

\section{2. $\mathrm{D} \varnothing$}

$\mathrm{D} \varnothing$ detector also has been submitted to many modifications and the figure 1 summarizes the main features.

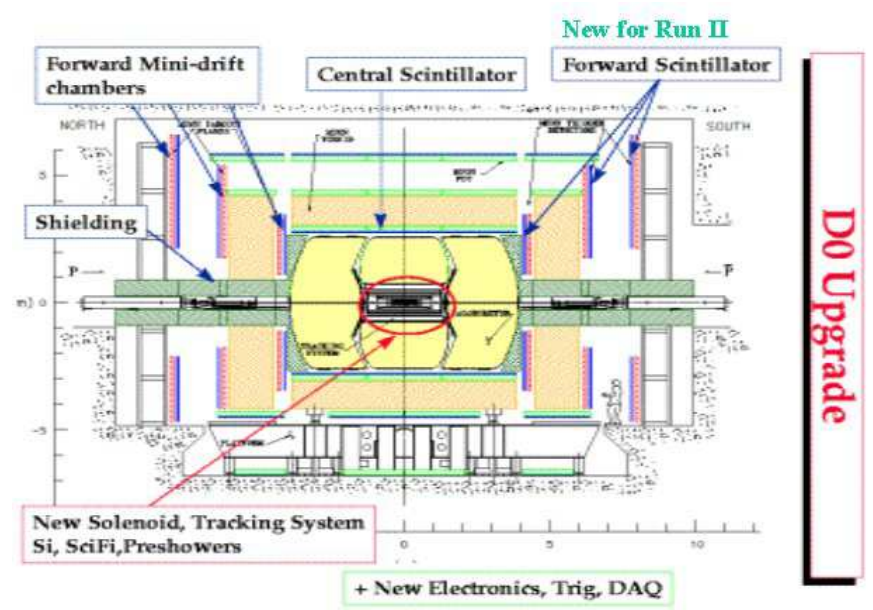

Figure 4. This figure shows the upgrade of the DØ .

Now we will describe the Forward Proton Detector for $\mathrm{D} \varnothing$.

\section{Forward Proton Detector}

The idea of the Forward Proton Detector is to cover experimentally a large number of topics which will be very important for the progress of the diffractive physics. Many details of FPD we can read in the reference [8].

The Forward Proton Detector consists of 18 Roman Pots arranged on both sides of the $D \varnothing$ detector as is shown in figure 5 where the Roman Pots are in the beam line of the Tevatron. We have two castles on the proton side indicated by $P 1$ and $P 2$ as is shown in figure 5 . The orientation is indicated by the additional letter $\mathrm{U}$ for up position, D for down position, I for inside position and $\mathrm{O}$ for outside position of the pots $(P 1 U, P 1 D, P 1 I, P 1 O$, same notation for $P 2$ ). On the anti-proton side we have two similar castles labeled by $A 1$ and $A 2$ followed by the indication of the position similar for the proton side. Two others half castles on the anti-proton side are the dipole magnet labeled $D 1$ and $D 2$. The approximated distances of the pots with respect to the interaction point (indicated by 0 on the scale) are shown.

\subsection{Some Physics Topics for the Run II at Tevatron}

With the FPD we intend to cover a good phase space region, in order to revisit some old and interesting results and create the conditions to observe new physics topics in diffraction. The combination of the Dipole and Quadrupole stations will allow us to get a good sample of data. On table 1 we show a comparison between the available data and the possible sample to get with FPD.

The topics of physics can be distributed by some topologies. In figure 6 we show several possibilities.

Some of the topics that can be studied we have:

\subsubsection{Low and High $|t|$ Elastic Scattering}

The acceptance of FPD spectrometer for low and high $t$ will allow us to extract elastic scattering events for both physical region. The measurements of elastic cross sections give a direct connection to the total cross section via Optical Theorem. It is important to know the elastic slope of the differential cross section. The value of the 


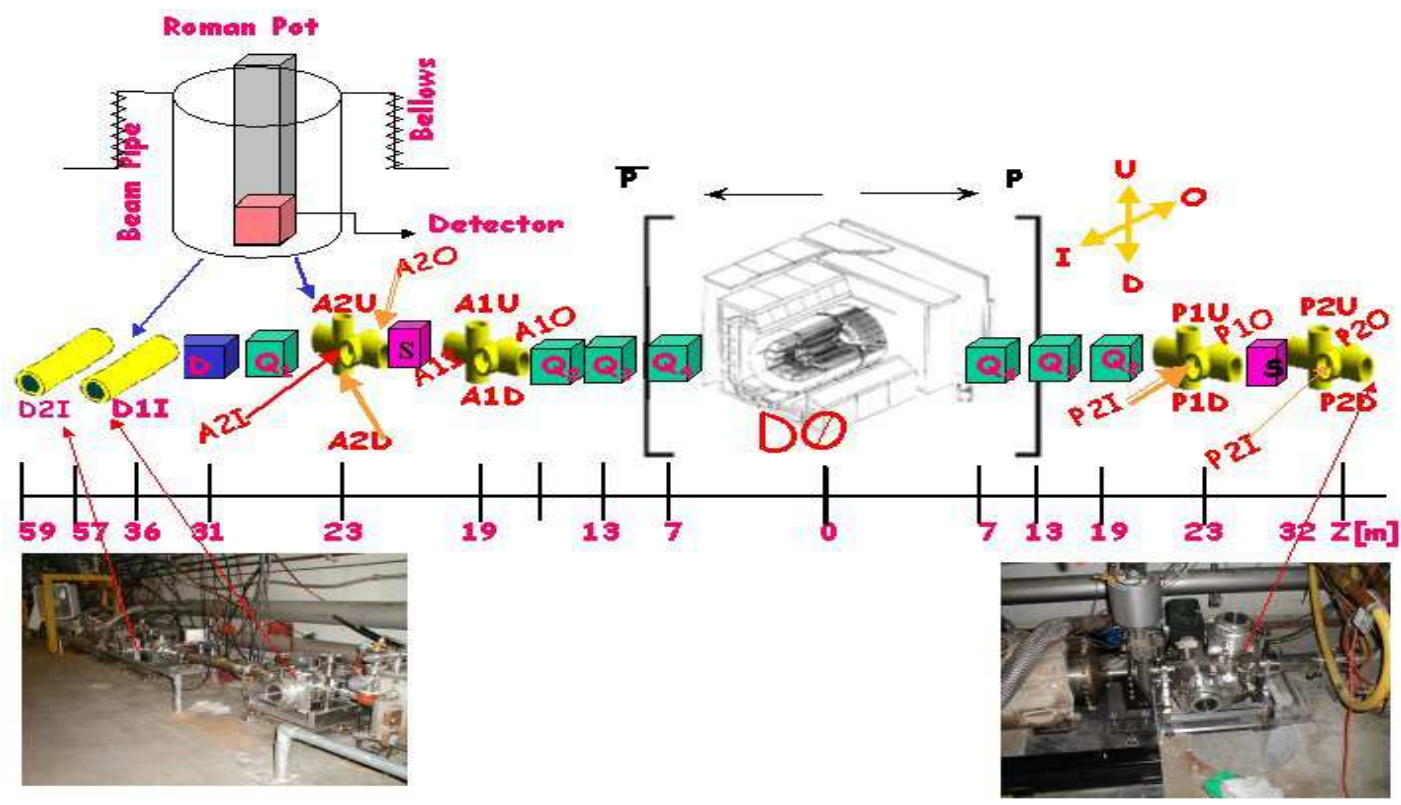

Figure 5. This figure shows the FPD in the beam line of the Tevatron, in both sides of the DØ detector.

Table 1

This table shows a comparison between what we have in the present and what is our expectation using FPD at the Run II of the Tevatron.

\begin{tabular}{||c|c|c||}
\hline \hline Experiment & Dijet Events & $E_{T}[\mathrm{GeV}]$ \\
\hline \hline UA8 & 100 & 8 \\
\hline HERA & Hundreds & 5 \\
\hline CDF & Thousands & 10 \\
\hline \multirow{3}{*}{ D $/$ FPD } & 500,000 & 15 \\
\cline { 2 - 3 } & 150,000 & 20 \\
\cline { 2 - 3 } & 15,000 & 30 \\
\hline \hline
\end{tabular}

slope for general differential cross sections characterizes a specific process which can be associated to a particular production (e.g. resonance production). We can see in figure 6 the association of the expected lego plot of pseudo-rapidity versus azimuthal angle showing a gap between the scattered proton and anti-proton scattered.

\subsubsection{Total cross section}

The results from Tevatron [9] experiments are not compatible between them if we extrapolate to higher energies. It would be very important to have another measurement at these energies. It is important to know the behavior of the cross sections with energy since we have a behavior predicted by the Froissard bound. After the Tevatron only the LHC will offer a new opportunity to make these measurements. The measurements made at the Tevatron will guide the future measurements at higher energies.

\subsubsection{Inclusive single diffraction}

The inclusive single diffraction has many subjects associated with it. Particularly for the Tevatron detectors, the Diffractive mass available, for single diffraction events, $M_{x}=450 \mathrm{GeV}$, makes the extraction of heavy flavor physics comfortable. Inclusive single diffraction has been a good laboratory for several problems in diffraction physics. We intend to use it to study jets and to calculate ratios between cross sections of different processes.

\subsubsection{Diffractive Jet production}

Jets have been largely studied by QCD. The discovered of diffractively produced jets [2] by UA8 collaboration was very important for diffractive physics. This was the main starting point for 


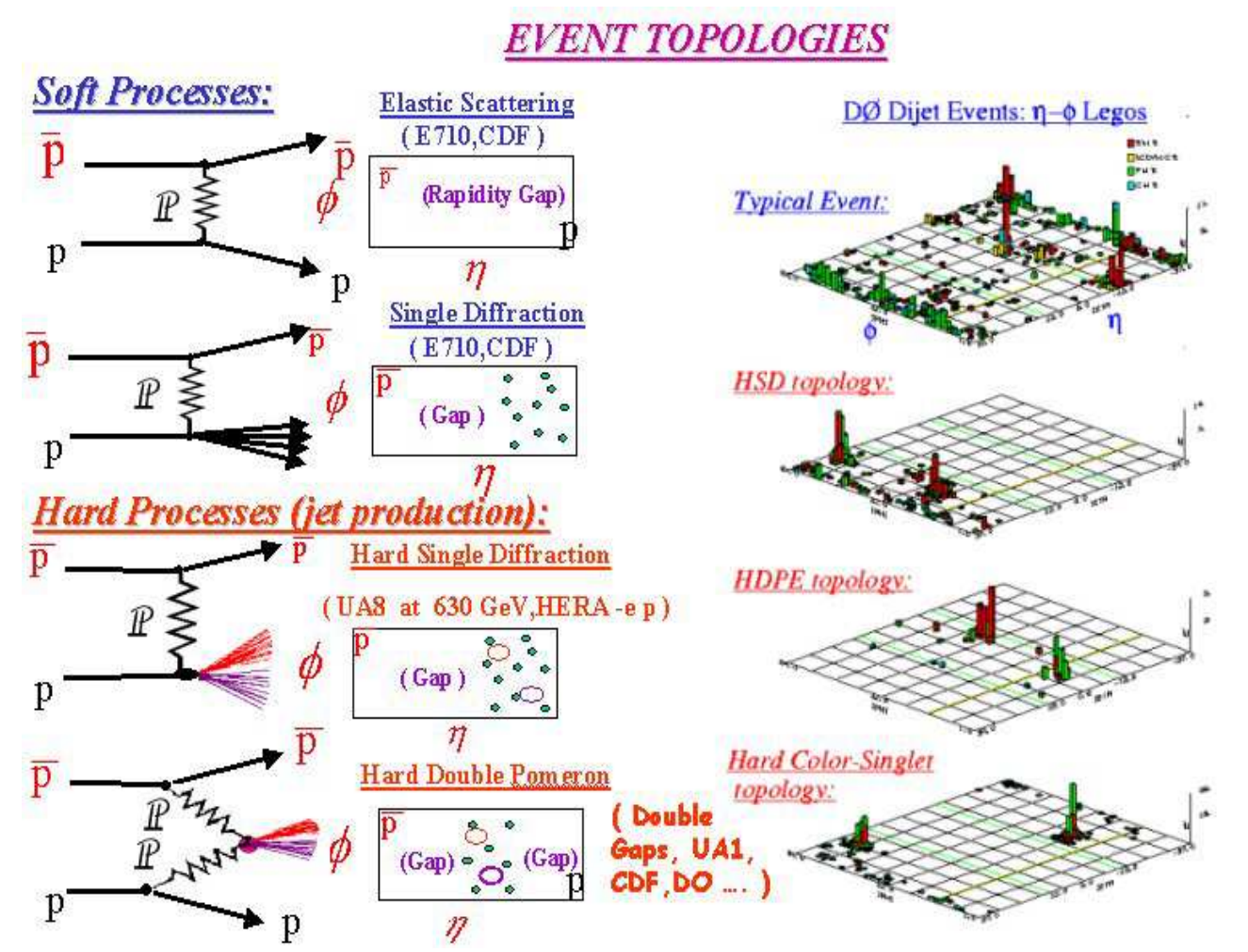

Figure 6. This figure shows the possible topologies to be studied with FPD in D $\varnothing$ detector. For each topology we have a corresponding lego plot in pseudo-rapidity versus azimuthal angle. We show also the lego plots corresponding to three topologies: the hard single diffraction (HSD), the hard double Pomeron Exchange (HDPE) and the Hard Color-Singlet extracted from DØ dijet events.

hard diffraction. It is expected that single and double jet diffractive production be exhaustively studied in the near future with FPD, making a distinction to the jets produced by the flux of color interactions. As we see in table 1 it is possible to get dijet events with transverse energy $E_{T}$ higher in run II than in run I.

\subsubsection{Hard Double Pomeron Exchange}

Due to the interesting topology, exciting physics topics, double Pomeron exchange has been largely discussed as the process for many different types of production [10]. An advantage of the large Diffractive mass produced at the Tevatron, in this case $M_{x} \simeq 100 \mathrm{GeV}$, is the possibility to study by direct observation the Pomeron $x$ Pomeron interactions and the associated physics. The instrumentation proposed by FPD/ D $\varnothing$ is appropriated to face the challenges of the double Pomeron mechanism, and to produce several objects not yet observed. Figure 6 shows the double Pomeron exchange graph and the gaps on its corresponding lego plot. Other topics like Glueballs, Centauros and Higgs can be exploited also in this topology.

\subsubsection{Diffractive Heavy Flavor Production}

Heavy Flavor physics has been extensively studied in high $p_{t}$ physics. Because experimental results are rare for heavy flavor diffractively produced, there was not enough attention to this physics. For lack of adequate instrumentation we have cross sections without a trigger separation for diffractive and non-diffractive events. Following the flavour of quarks we can split the studies of diffractive heavy flavor production, (i) the 
Charm, (ii) the Bottom and the (iii) Top Physics. Each one has some particularities to be taken into account in the diffractive production 11].

Heavy flavor physics has been considered almost as a high $p_{t}$ physics only. Diffractive heavy flavor production has not been sufficiently studied. This is due mainly to the absence of experimental results in this area as a consequence of lack of adequate instrumentation to observe diffractive production of heavy flavor.

\subsubsection{Diffractive $\mathrm{W} / \mathrm{Z}$ boson production}

The present results from diffractively produced $\mathrm{W}$ and $\mathrm{Z}$ bosons are not satisfactory, we need more statistics for these events. This is important to better understand these processes, in order to have a comparison with the current boson production. Both CDF 12] and DØ have made progress, and the current results are motivating both collaborations to proceed with new measurements.

\subsubsection{Diffractive Structure Functions}

The study of Diffractive structure functions at the Tevatron would allow a comparison with the existing Hera results. To understand the structure of the Pomeron one must know its structure function. This type of study has to be pursued exhaustively to get better accurate events and to have a clear interpretation of the Pomeron. One should know how important is the gluon and the quark component of the Pomeron. The universality of the Pomeron components is important in order to have more clear idea of this object. Practically all topics depend of the knowledge of the structure function of the Pomeron. Many theoretical developments depend on the experimental results for diffractive structure function. It is important to build a good sample of events to study the diffractive structure functions in $\bar{p} p$ at the energy of the Tevatron.

\subsubsection{Glueballs, Centauros and Higgs}

Since the origin of QCD, Glueballs has been studied by theoreticians and experimentalists. However, we do not have a significant progress in this subject. We need more experiments dedicated to the discover of glueballs without ambiguity with quark anti-quark competitive states. The family of glueballs is big. Table 2 shows the glueballs (oddballs are also shown). Oddballs should have the priority to be examined due to the fact that they do not have competition with current $q \bar{q}$ states, mesons, and the $q q q$ states, (baryons) with the same quantum numbers. It is difficult to separate the common hadrons from the glueballs when they appear in the same physical region. Glueballs can be produced also in double Pomeron Exchange topology. We believe that glueballs are largely produced by this mechanism.

Another topic is the production of Centauros, which were never observed in accelerator particle physics. These objects were discovered in Cosmic Ray Physics as events with several unusual characteristics, like the production of large multiplicity charged particles accompanied by very few photons. For example, as many as 100 charged particles and no more than $3 \pi^{0}$ [13]. We have enough energy at the Tevatron to produce Centauros. Since our diffractive mass is significantly high, we can produce them diffractively. The good calorimetry of the $\mathrm{D} \varnothing$ detector can be very useful to observe the absence of electromagnetic activity.

Higgs is one of the exciting subject in the next Run II. It is not excluded that they are produced also diffractively. We have two recently studies giving by references [10, showing the possibility of Higgs production by double Pomeron mechanism.

3.1.10. Correlations between $\eta, \mathbf{t}, M_{x}, \mathbf{b}, \xi$, $\mathbf{x}, E_{T}, .$.

During the study of any subject in diffractive physics it would be important to know the behavior of the data with respect to a few number of kinematics variables and their correlation among themselves. The kinematical variables are: the pseudo-rapidity $\eta$ defined by

$\eta=-\ln \left(\tan \frac{\theta}{2}\right)$,

$t$ is the transferred momentum between the proton beam and the scattered proton defined by $t=\left(P_{\text {Beam }}-P_{\text {Scattered }}\right)^{2} ; M_{x}=\sqrt{\xi} \sqrt{s}$ is the diffractive mass which is 450 . $\mathrm{GeV}$ for single diffraction and 100. GeV for double Pomeron exchange at the energies of the Tevatron $(\sqrt{2} \mathrm{TeV})$; 
Table 2

This table shows possible glueball state configurations with the mass and the quantum numbers for each one.

\begin{tabular}{||c|c|c|c|c|c|c|c||}
\hline \hline \multicolumn{9}{||c||}{ Glueballs and Oddballs } \\
\hline \multirow{2}{*}{$J^{P C}$} & \multirow{2}{*}{$(q \bar{q})$} & \multirow{2}{*}{$2 \mathrm{~g}$} & \multirow{2}{*}{$3 \mathrm{~g}$} & \multirow{2}{*}{ ODD } & \multicolumn{3}{|c||}{ MASS $(\mathrm{GeV})$} \\
\cline { 6 - 9 } & & & & & {$[14$} & $\| 15]$ & {$[16]$} \\
\hline \hline $0^{++}$ & YES & YES & YES & NO & 1.58 & $1.73 \pm 0.13$ & $1.74 \pm 0.05$ \\
\hline $0^{+-}$ & NO & NO & YES & YES & & & \\
\hline $0^{-+}$ & YES & YES & YES & NO & & & \\
\hline $0^{--}$ & NO & NO & YES & YES & 2.56 & $2.59 \pm 0.17$ & $2.37 \pm 0.27$ \\
\hline $1^{++}$ & YES & YES & YES & NO & & & \\
\hline $1^{+-}$ & YES & NO & YES & NO & & & \\
\hline $1^{-+}$ & NO & YES & YES & YES & & & \\
\hline $1^{--}$ & YES & NO & YES & NO & 3.49 & $3.85 \pm 0.24$ & \\
\hline $2^{++}$ & YES & YES & YES & NO & 2.59 & $2.40 \pm 0.15$ & $2.47 \pm 0.08$ \\
\hline $2^{+-}$ & NO & NO & YES & YES & & & \\
\hline $2^{-+}$ & YES & YES & YES & NO & 3.03 & $3.1 \pm 0.18$ & $3.37 \pm 0.31$ \\
\hline $2^{--}$ & YES & NO & YES & NO & 3.71 & $3.93 \pm 0.23$ & \\
\hline $3^{++}$ & YES & YES & YES & NO & 3.58 & $3.69 \pm 0.22$ & $4.3 \pm 0.34$ \\
\hline $3^{+-}$ & YES & NO & YES & NO & & & \\
\hline $3^{-+}$ & NO & YES & YES & YES & & & \\
\hline $3^{--}$ & YES & NO & YES & NO & 4.03 & $4.13 \pm 0.29$ & \\
\hline \hline
\end{tabular}

$b$ is the measured slope of the differential cross section, which can be selected globally or for a particular region of the invariant mass produced diffractively $\left(\frac{d \sigma}{d t} \propto e^{-b\left(M_{x}\right) t}\right) ; \xi=1-x_{p}=\frac{\Delta P}{P}$ is the fraction of the momentum of the proton carried by the Pomeron; $x_{p} \geq 0.95$ is the fraction of the momentum of the proton carried by the scattered proton; and $E_{T}=$ is the transverse energy of the jet produced by hard diffraction; $\theta$ is the polar angle of the scattering and $\phi$, the corresponding azimuthal angle. The distributions of these kinematics variables are a phenomenological source of investigation of the possible hidden dynamics of the diffraction. The lego plot $\eta$ the pseudo-rapidity, versus $\phi$ it is an example of this type of study. The rapidity gap still is a good signature for diffractive events.

\subsection{Roman Pots}

The design of the Roman Pots of D $\varnothing$ FPD is shown in figure 7. They were built by LNLS/Brazil (Laboratório Nacional de Luz Syn- chroton) as part of our regional collaboration ( LAFEX/CBPF -Centro Brasileiro de Pesquisas Físicas; UFBA -Universidade Federal da Bahia; UFRJ -Universidade Federal do Rio de Janeiro; UERJ -Universidade Estadual do Rio de Janeiro; IFT/UNESP -Universidade Estadual Paulista; and LNLS). During two years we studied many options for the castle and detectors. The castle was made using 316L steel following the technical specifications to obtain the high vacuum of the Tevatron. The combination of the four views, quadrupole stations as is shown in figure 0 and the dipole stations give the possibility to cover more phase space and to get a better acceptance. In figure 7 we show the castle, the support of the castle and the pot itself made by NIKHEF laboratory. In order to get the best pot performance the design decision of the pot window of the pot was submitted to the finite element analysis. 150 microns foil with elliptical cutout gave the best result. 


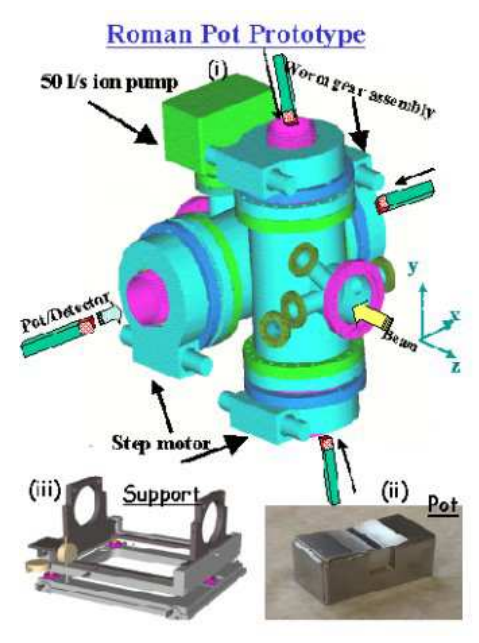

Figure 7. This figure shows the (i) castle, the (ii) support of the castle and the pot itself. The figure shows all main parts of the castle, four directions; in each one a pot is being inserted. The pots moving to be approximated of the beam using step motors. These three parts constitute the mechanical part of the Roman Pots Stations.

\subsection{The Detectors}

For the detectors we choose the 800 microns scintillator fibers as is shown in figure 8. In this figure 8 we see the frame of the scintillating fibers and 6 planes $\mathrm{X} \mathrm{X}, \mathrm{U} \mathrm{U}^{\prime}$ and $\mathrm{V} \mathrm{V}^{\prime}$ which compose one unity or one detector to be inserted in the pots. The scintillating fibers are glued to clear fibers which guide the signal up to the multi-anode photomultipliers (MAPMT H6568 from Hamamatsu). We have 16 channels per plane X X' and 20 channels/plane U U', V $\mathrm{V}^{\prime}$, giving a total of 112 channels per detector and 2016 channels in total. Studies about the signals, efficiency and resolution have been made. Scintillating fibers are the best option for our detectors among many other possible technologies. The frame is made of ordinary plastic. The theoretical resolution is 80 microns. The acceptance of our detectors has been studied in several views.

The geometrical acceptance and the pot posi-

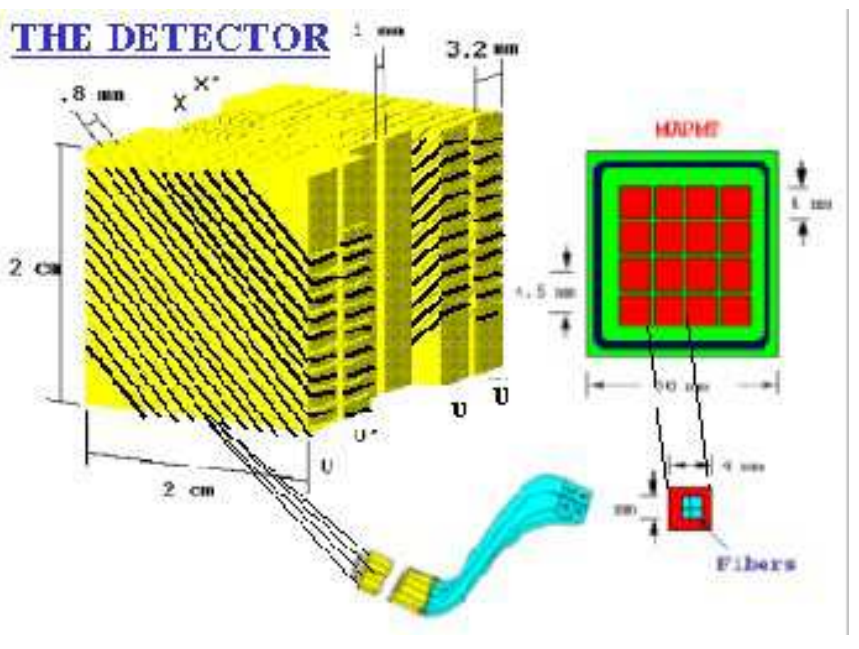

Figure 8. This figure shows the six planes (u u', v $v^{\prime}, x^{\prime}$ ') of the detectors of the FPD spectrometer and the MAPMT. Each form scintillator fibers is connected in the figure join to a cell of the MAPMT.

tion acceptance is given in figure 9 .

\section{Conclusion}

The diffractive physics at Tevatron in the next Run II will be very exciting. Practically all possible subjects can be studied with the data obtained by CDF and D $\varnothing$ FPD. We have shown how interesting are the upgrades including Tevatron, Detectors and subdetectors for diffractive physics.

The main goal of the experiments in diffractive physics is the hard diffraction. Many theoretical and phenomenological progress has been done without a corresponding set of data allowing the comparisons. New data is urgently needed to establish limits and to guide our imagination.

The primary physics goal of the FPD is to measure hard diffraction, obtaining new data, for the progress of the diffractive physics. This is an opportunity to clarify many hypothesis made theoretically as well as to test the present models for 

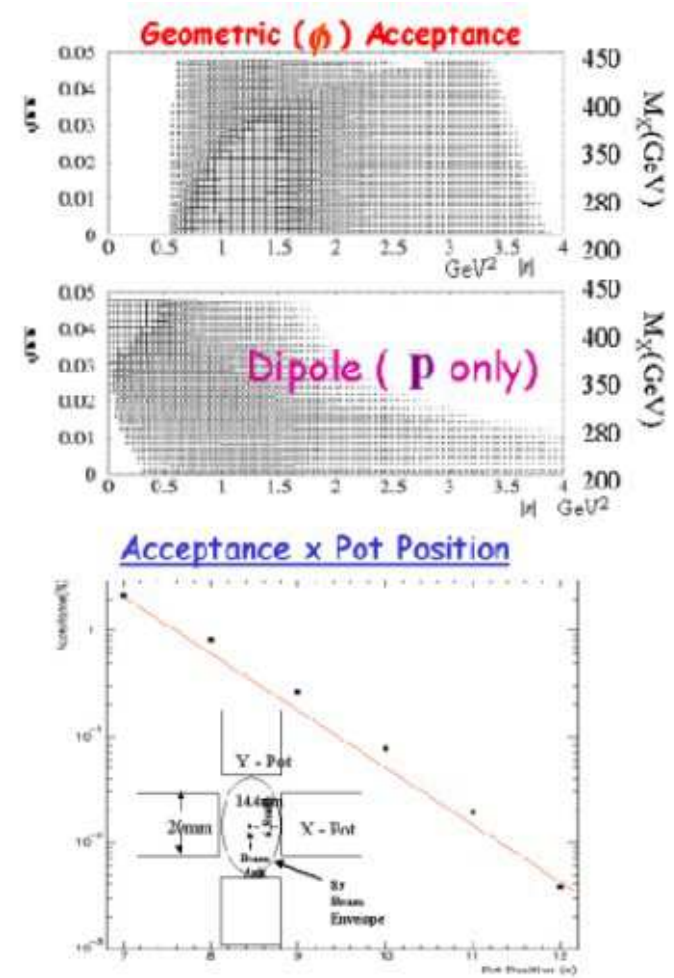

Figure 9. This figure shows the acceptance $\xi$ t for both dipole and quadrupole stations. The figure shows also the acceptance versus the pot position. We have an idea of the acceptance variation moving the pot to be as near as possible of the beans.

diffraction. The data will also give the possibility to have a good comparison with the Hera diffractive physics production of last years in order to test the universality of the Pomeron. It will also be possible to use the FPD to reduce uncertainties on the luminosity for all $\mathrm{D} \emptyset$ physics processes. The double Pomeron exchange and the physics that can be done with this topology is one of the very important subject of this next Run II of the Tevatron. It is not excluded as we called attention, that Centauros and Higgs [10] can be produced diffractively in the double Pomeron topology.
We will obtain results which will improve old measurements at lower energies and, in some cases, can contribute for clarifying existing results as is the case of the total cross sections in Tevatron energy. We gave a list of possible topics to be investigated in both hard and soft diffraction.

Our schedule to start the data acquisition is the same of the D $\varnothing$ Detector i.e., at the beginning of the year 2001. Finally the diffractive physics results of DØFPD, will be very important for future projects at LHC, since it is expected that Diffractive Physics becomes more important when the energy increases.

I would like to thank the organizing committee of the International Workshop on Diffraction in High-Energy Physics, Diffraction 2000, and in particular Prof. R. Fiore, for having invited me to give this talk, giving me the opportunity to participate to this interesting Workshop. All the work behind this paper was done by our colleagues from $\mathrm{CDF}$ and $\mathrm{D} \varnothing$ collaboration. We thank the organizing committee of the Diffraction 2000 and FAPERJ for partial financial support.

\section{REFERENCES}

1. For a nice historical summary about Diffraction see: Enrico Predazzi, Opening Talk in this Workshop.

2. A. Brandt et al., UA8 Collaboration, Nucl. Inst. Meth. A327, 412 (1993); id. , Phys. Lett. B297, 93 (1993); ibid. , HEP-Ex9709015, 1997, submitted to PLB.

3. ZEUS collaboration, M. Derrick et al., Z. Phys. C65, 379 (1995); H1 collaboration, T. Ahmed et al., Nucl. Phys. B439, 471 (1995); ZEUS collaboration, M. Derrick et al. Z. Phys. C68, 569 (1995); H1 collaboration, T. Ahmed et al. Phys. Lett. B348, 681 (1995); and talks presented in this Workshop.

4. E.M. Gregores, T.L. Lungov and S.F. Novaes, A. Santoro, Proceedings of Hadron 2000, S. Paulo, Brazil (2000).

5. Instead to write again the results from DØ I would like to give a reference about my talk in this conference DØ Hard Diffraction, which was presented in several conferences by many colleagues from D $\varnothing$ collaboration. All infor- 
mation are in Hard Single Diffraction at $\sqrt{s}$ $=630$ and $1800 \mathrm{GeV}$, DØ Collaboration, B. Abbot et al.; Fermilab-Pub-99/375-E; hepex/9912061.

6. See the talk of this Workshop of Giorgio Matthiae in this proceedings.

7. See the talk of this Workshop of K. Goulianos in this proceedings.

8. A. Brandt et al., A Forward Proton Detector, Fermilab-Pub-97/377, 1997.

9. C. Avila, Proceedings of LISHEP98; Thesis: Measurement the proton - antiproton total cross section at center of mass Energy of $1800 \mathrm{GeV}$, Cornell University 1997 and references therein; C. Avilea et al., Phys. Lett. B234, 158 (1990); ibid. , Phys. Rev. Lett. 68, 2433 (1992); ibid. , Phys. Rev. 50, 5550 (1994); ibid. , Phys. Lett. B234, 158 (1990); ibid., Nucl. Inst. Meth. A360, 80 (1995).

10. See for example for Higgs production the paper of D. Kharzeev and E. Levin, Soft Double-Diffractive Higgs Production at Hadron Collider, Fermilab-Pub-00/035-T; BNL-NT-00/14; hep-ph/0005311 (2000); and Can the Higgs be seen in rapidity gap events at the Tevatron or the LHC?, V.A. Khoze, A.D. Martin and M.G. Ryskin, DTP/00/08; hep-ex/0002072 (2000).

11. E.L. Berger, J.C. Collins, D.E. Soper, G. Sterman, Nucl. Phys. B286, 704 (1987); A. Kerman and G. Van Dalen, Phys. Rep. 106, 297 (1984).

12. F. Abe et al., CDF Collaboration, Phys. Rev. Lett. 78, 2698 (1997).

13. F. Halzen, FELIX: The Cosmic Ray Connection, Felix Home Page http:// felix.web.cern.ch/FELIX/Physics/paperi.html, Brazil-Japan Collaboration, Proceedings of the 21st. Int. Conf., Adelaide, Australia, vol. 8, 259 (1990); C.M.G. Lattes, Y. Fujimoto and S. Hasegawa, Phys. Rep. 65, 151 1980; C. E. Navia et al., Phys. Rev D40, 2898 (1989).

14. A.B. Kaidalov and Yu.A. Simonov, Glueball masses and Pomeron Trajectory in nonperturbative QCD approach, hep-ph/9912434 (1999).

15. C. Morningstar, M. Peardor, Nucl. Phys.
B63A-C, 1022 (1998); Phys. Rev. D60, 034509 (1999).

16. M. Teper, Glueball masses and other physical properties of $S U(N)$ gauge theories in $D=3+1$ : a review of lattice results for theorists, hep-th/981287 (1998). 